\title{
Endoscopic therapy of gallstones ileus
}

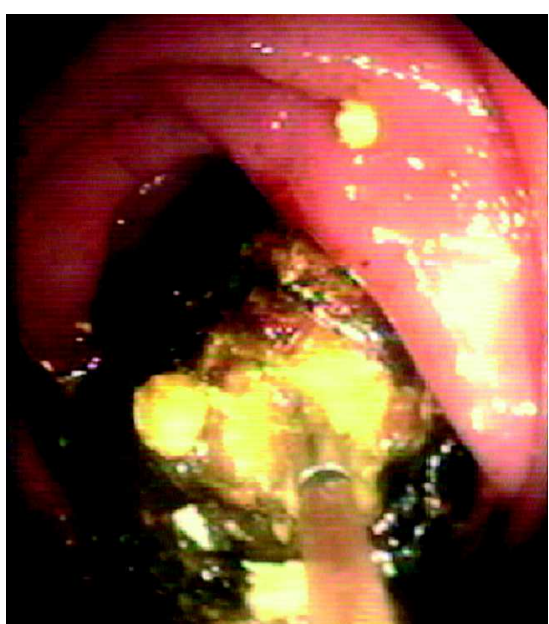

Fig. 1 Endoscopic view of the obstructing gallstone.

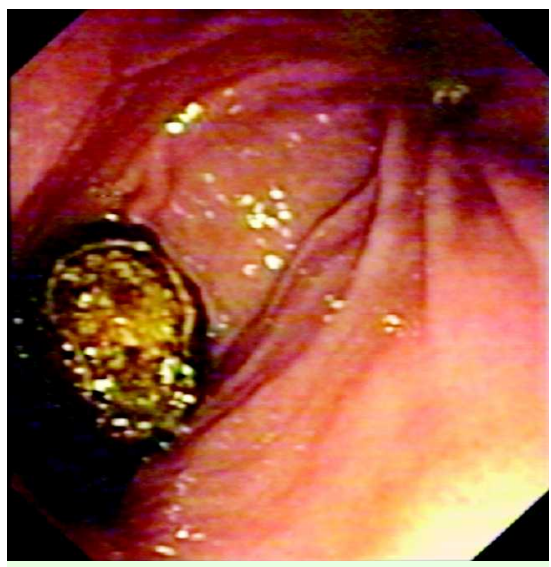

Fig. 3 Endoscopic view of the gallstone in the antrum.

Gallstone ileus is a rare clinical complication of gallstone disease and is primarily treated surgically. We describe three cases of gallstone ileus, including the first report of successful treatment of jejunal gallstone ileus by endoscopically applied electrohydraulic lithotripsy (EHL).

A 67-year-old woman was admitted to hospital with epigastric pain. Ultrasonography showed gallbladder stones and air in the biliary tree. Intestinoscopy detected a stone in the jejunum that occluded the bowel lumen ( $\bullet$ Fig. 1). After unsuccessful attempted extraction of the stone using a Dormia basket, EHL was performed (Walz Lithotron EL 23 generator; Walz Electronic Inc., Rohrdorf, Germany) using a 4.5 Ch EHL probe (Olympus Optical Co. GmbH, Hamburg, Germany), which disintegrated the stone. After this

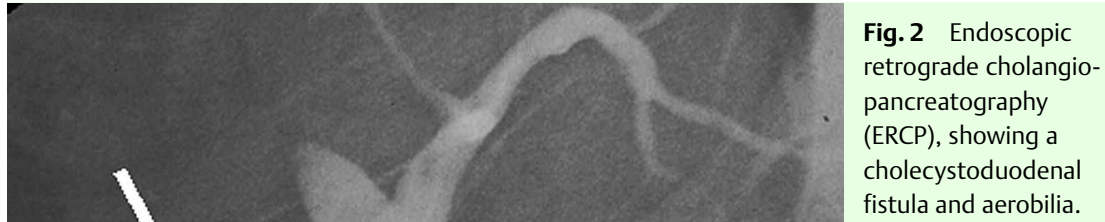

had been done, duodenoscopy and endoscopic retrograde cholangiopancreatography (ERCP) showed a cholecystoduodenal fistula ( $\bullet$ Fig. 2) and the patient underwent laparoscopic cholecystectomy.

A 71-year-old woman was admitted with postprandial bilious vomiting and abdominal pain. Esophagogastroduodenoscopy showed an obstructing gallstone in the bulbus duodeni. Extraction using a Dormia basket failed due to the size of the gallstone. EHL was performed and the stone was fragmented.

A 69-year-old woman was admitted with upper abdominal pain. Gastroscopy revealed ulcers in the antrum, one of which represented perforation by the gallstone into the antrum, whereby the stone located in the antrum did not cause an ileus ( $\bullet$ Fig. 3). ERCP demonstrated reflux of contrast medium into the antrum. Mechanical lithotripsy was successfully performed.
Cholecystitis often precedes the perforation of the gallstone into the gut forming a cholecystoenteric fistula. The main clinical presentation is gastric outlet obstruction of the duodenal bulb (Bouveret's syndrome). Kasahara et al. reported spontaneous passage of gallstones in only eight of 112 patients in Japan [1]. Demonstration of aerobilia by computed tomography (CT) scan is widely accepted as the preferred imaging method for determining the site of obstruction. Gallstone ileus is normally treated by laparotomy and enterolithotomy [2]. Because of the relatively high morbidity and mortality, alternative procedures to surgery have been suggested. Mechanical lithotripsy [3], EHL [4], and intraluminal laserlithotripsy [5] have been successfully applied. Here we demonstrate the usefulness of therapeutic endoscopically guided intraluminal lithotripsy. 
Endoscopy_UCTN_Code_TTT_1AR_2AH

\section{H. Schwacha ${ }^{1}$, H. E. Blum ${ }^{1}$,}

P. Hafkemeyer ${ }^{2}$

1 Department of Medicine II, University Hospital Freiburg, Freiburg, Germany

2 Department of Medicine, Kreiskrankenhaus Emmendingen, Emmendingen,

Germany

\section{References}

1 Kasahara Y, Umemura H, Shiraha S. Gallstone ileus: review of 112 patients in the Japanese literature. Am J Surg 1980; 140: 437-440

2 Reisner RM, Cohen JR. Gallstone ileus: a review of 1001 reported cases. Ann Surg 1994; 60: 441 - 446

3 Lübbers H, Mahlke R, Lankisch PG. Gallstone ileus: endoscopic removal of a gallstone obstructing the upper jejunum. J Intern Med 1999; 246: $593-597$

4 Moriai T, Hasegawa T, Fuzita $M$ et al. Successful removal of massive intragastric gallstones by endoscopic electrohydraulic lithotripsy and mechanical lithotripsy. Am J Gastroenterol 1991; 86: 627-629

5 Maiss J, Hochberger J, Muehldorfer S et al. Successful treatment of Bouveret's syndrome by endoscopic laserlithotripsy. Endoscopy 1999; 31: 4-5
Bibliography

DOI $10.1055 / \mathrm{s}-2008-1077385$

Endoscopy 2008; 40: E185-E186

(c) Georg Thieme Verlag KG Stuttgart · New York . ISSN 0013-726X

\section{Corresponding author}

\section{P. Hafkemeyer, MD}

Kreiskrankenhaus Emmendingen

Gartenstaße 44

79312 Emmendingen

Germany

Fax: +49-7641-4542269

phafkemeyer@gmx.net 